\title{
La demolición a gran escala como técnica de intervención urbana. La apertura de la Avenida de la Constitución de Sevilla (1906-1927)
}

\author{
Demolition on a large-scale basis as a technique for urban intervention. \\ The opening of "Avenida de la Constitución" in Seville (1906-1927)
}

\author{
J. Tejido Jiménez ${ }^{(*)}$
}

\section{RESUMEN}

La apertura de la Avenida de la Constitución de Sevilla es una intervención urbana del primer tercio del siglo xx, heredera de los planteamientos de Haussmann en París, y con semejanzas a la creación de la Gran Vía madrileña. Se realizó en un momento en el que aún no estaba formada la conciencia del valor patrimonial de los tejidos históricos, por lo que se basó en el borrado a gran escala de las tramas preexistentes. Su ejecución precisó, entre otras demoliciones, del derribo de dos edificios notables del siglo xvi, el antiguo Colegio de Santa María de Jesús - primera sede de la Universidad de Sevilla- y el antiguo Colegio dominico de Santo Tomás. Se confiaba en que la sección de la apertura y el porte de las nuevas arquitecturas que surgirían a continuación construirían la deseada Avenida, de la que todavía vive el acceso sur al centro monumental de Sevilla.

Palabras clave: Apertura; ensanche; avenida; Sevilla; patrimonio arquitectónico; Colegio de Santa María de Jesús; Colegio de Santo Tomás.

\section{ABSTRACT}

Developed over the first third of 2oth century, the opening up of "Avenida de la Constitucion" in Seville inherits some of Haussmann's urban strategies carried out in Paris some time before, and presents clear similarities with the creation of Gran Via in Madrid. It took place at a time when historic urban fabric protection principles were not yet settled upon collective consciousness, thus it was mainly based on a large-scale deletion of the pre-existing plot. Amongst others, two major 16th century architecture samples, such as former Santa María de Jesús College (first home to the University of Seville) and Santo Tomás College, were teared down to enable this urban operation. Confidence upon the wider crosssection benefits and the new architecture style it would bring along was at the core of this envisioning, generating a space that still keeps its role as main gateway to the historic centre of Seville.

Keywords: Opening; widening; avenue; Seville; architectural heritage; Santa María de Jesús College; Santo Tomás College.

(*) Escuela Técnica Superior de Arquitectura - Universidad de Sevilla (España). Persona de contacto/Corresponding author: jtejido@us.es (J. Tejido Jiménez)

Cómo citar este artículo/Citation: Tejido Jiménez, J. (2016). La demolición a gran escala como técnica de intervención urbana. La apertura de la Avenida de la Constitución de Sevilla (1906-1927). Informes de la Construcción, 68(541): e137, doi: http://dx.doi. org/10.3989/ic.15.045.

Licencia / License: Salvo indicación contraria, todos los contenidos de la edición electrónica de Informes de la Construcción se distribuyen bajo una licencia de uso y distribución Creative Commons Reconocimiento no Comercial 3.o. España (cc-by-nc). 


\section{INTRODUCCIÓN}

En 2006 se cumplió el primer centenario de la aprobación del proyecto de apertura de la Avenida de la Constitución de Sevilla, trazado en 1906 por el arquitecto municipal José Sáez López (1). Su ejecución se llevó a cabo lentamente, por tramos, completándose el último de ellos en 1927, en los momentos previos a la Exposición Iberoamericana de 1929 (2). La Avenida es, por lo tanto, un espacio moderno abierto en el corazón del casco histórico mediante una potente intervención urbana, heredera de la cultura decimonónica que propugnaba la creación de grandes ejes sobre las ciudades antiguas. Tiene su principal referente en las operaciones de Haussmann en París y es coetánea a la creación de la Gran Vía madrileña y otras grandes aperturas españolas (3). La aplicación de estos criterios de transformación de la ciudad obligó a sacrificar algunos edificios de valor y espacios urbanos consolidados, que en la actualidad, con la consideración contemporánea sobre el patrimonio arquitectónico, habrían tenido un tratamiento muy diferente.

\section{INSTRUMENTOS DE LA INTERVENCIÓN}

La apertura se planteó en una etapa en la que aún no estaba formada la conciencia del valor de los tejidos históricos, por lo que estuvo basada en el borrado a gran escala de las tramas heredadas, implicando la demolición sistemática de las construcciones a las que afectaba el trazado. Tuvo una considerable complejidad de ejecución, ya que las peculiaridades de los diferentes segmentos sobre los que se actuó exigieron diversidad de herramientas y procedimientos legales, empleándose según los casos la expropiación forzosa, la adquisición pactada, el convenio con los propietarios y la permuta de edificios. Todos estos procesos confluían en la demolición de las áreas seleccionadas, constituyendo la técnica que se empleó durante las primeras décadas del siglo xx para la intervención en la ciudad. En lo relativo a los instrumentos urbanísticos, la creación de la Avenida fue una actuación mixta en la que se combinó la modificación de alineaciones con la apertura directa a través de manzanas consolidadas. En el tramo situado al norte de la Catedral -la antigua calle Génova- el ensanche del espacio público se produjo mediante drásticos retranqueos de edificación, con operaciones de demolición individuales extendidas en el tiempo. Se realizaron acomodaciones de casas a las líneas aprobadas, lo que exigía la demolición de sus crujías delanteras y obligaba a los propietarios a reedificar nuevas fachadas, circunstancia que se aprovechó para actualizar los lenguajes arquitectónicos del sector intervenido, gran parte del cual se reparceló en lotes de mayor tamaño, en los que la arquitectura regionalista encontró un escaparate adecuado a sus realizaciones (4). En muchos casos se enajenaba la totalidad de la casa afectada, pagando por ella el Ayuntamiento una cantidad negociada con la propiedad, se derribaba la edificación y se sacaba a subasta la nueva parcela obtenida, tras habérsele restado la cesión a la vía pública. En los tramos situados al sur de la Catedral, caracterizados por la presencia de dos grandes conjuntos del siglo xvi, el antiguo Colegio dominico de Santo Tomás y el antiguo Colegio de Santa María de Jesús (primera sede de la Universidad de Sevilla), se aplicó una intervención más violenta y ajena a la trama, abriéndose el nuevo viario literalmente a través de las manzanas (Figura 1).

La apertura no contó con un proyecto arquitectónico que acompañase al plan de demoliciones a gran escala que estaba previsto, de tal manera que fue la propia acción destructiva la que sustentó la rotundidad de la intervención. Podemos afirmar que existió únicamente proyecto de demolición, mantenido a lo largo de dos décadas por una firme voluntad municipal y social de llevarlo a término, en tanto que la arquitectura de la vía surgió por la sumatoria de las nuevas construcciones, reguladas por unas ordenanzas municipales que fijaban la altura de la edificación proporcionalmente a la anchura de la calle, por lo que se generó de forma automática un espacio público de mayor escala. Paradójicamente, la ausencia de una propuesta concreta de arquitectura para la Avenida facilitó la evolución interna de la operación a lo largo del tiempo, permitiendo reconsiderar su anchura durante su ejecución, cuestión que hubiese resultado más difícil de abordar en caso de haberse dispuesto de un proyecto arquitectónico unitario. La operación produjo un efecto dominó, según el cual la acción destructiva demandó sobre la marcha más demoliciones para rematar los encuentros de los extremos de la Avenida con los contactos urbanos correspondientes, al norte con la Plaza Nueva y al sur con los jardines de Cristina (5).

En la documentación técnica que se elaboró, los arquitectos municipales se limitaban a dibujar el perímetro de las manzanas afectadas, señalando los extremos de fachadas de las casas implicadas. La materia arquitectónica objeto de demolición no se dibujaba, por lo que en la mayoría de los casos no ha quedado constancia gráfica de los rasgos tipológicos que se estaban eliminando de la ciudad. Constituye una excepción a este procedimiento la aparición de la planta de Santo Tomás en el proyecto de apertura de 1906, ya que al haberse dedicado este edificio a usos militares tras la desamortización, contaba con planimetría del Ejército, que se incorporó al proyecto. Sin embargo, la planta de Santa María de Jesús -que se utilizó como Seminario Conciliar desde mediados del XIX- se desconoce. Para llegar a una hipótesis de su conformación debe recurrirse al ensamblaje de los fragmentos de su historiografía: el plano de Padura de 1891 (que sitúa aproximadamente el claustro principal), la parcela que ocupaba el Seminario antes de su demolición (dibujada en el expediente de apertura de la Avenida de 1906), la planta de la capilla existente, las plantas del antiguo vestíbulo colegial y su sacristía (6), una ampliación del Seminario realizada en 1863 por el Arzobispado (7), y los trazados descubiertos por las excavaciones arqueológicas realizadas en 2006-2007, asociadas a la instalación del transporte público «metrocentro», entre los que destacan un fragmento de muralla de la ciudad, trazas parciales de estancias del Colegio y la esquina de un segundo claustro del edificio (8). Con este material gráfico es posible trazar una hipótesis de la morfología de esta zona de Sevilla con anterioridad a la apertura de la Avenida (Figura 2).

\section{PROCESO DE LA APERTURA}

Desde mediados del siglo xIx se fue asentando entre los estamentos técnicos y políticos municipales la idea de que la conformación medieval de la ciudad necesitaba reformas que la dotasen de vías más amplias, que permitiesen mejorar su permeabilidad interna y su capacidad de conexión con las primeras periferias. A finales del xix se empezó a considerar que la modificación de alineaciones, con la que se había estado manipulando la trama heredada durante décadas, no era un instrumento suficiente para conseguir una recualificación urbana que respondiese a las claves del momento (9). Se acometieron acciones más enérgicas, consistentes no sólo en retranquear las alineaciones de los edificios, sino en abrir nuevas calles a través de tejidos consolidados. Términos como vialidad, higiene o ventilación, entendidos como cualidades 

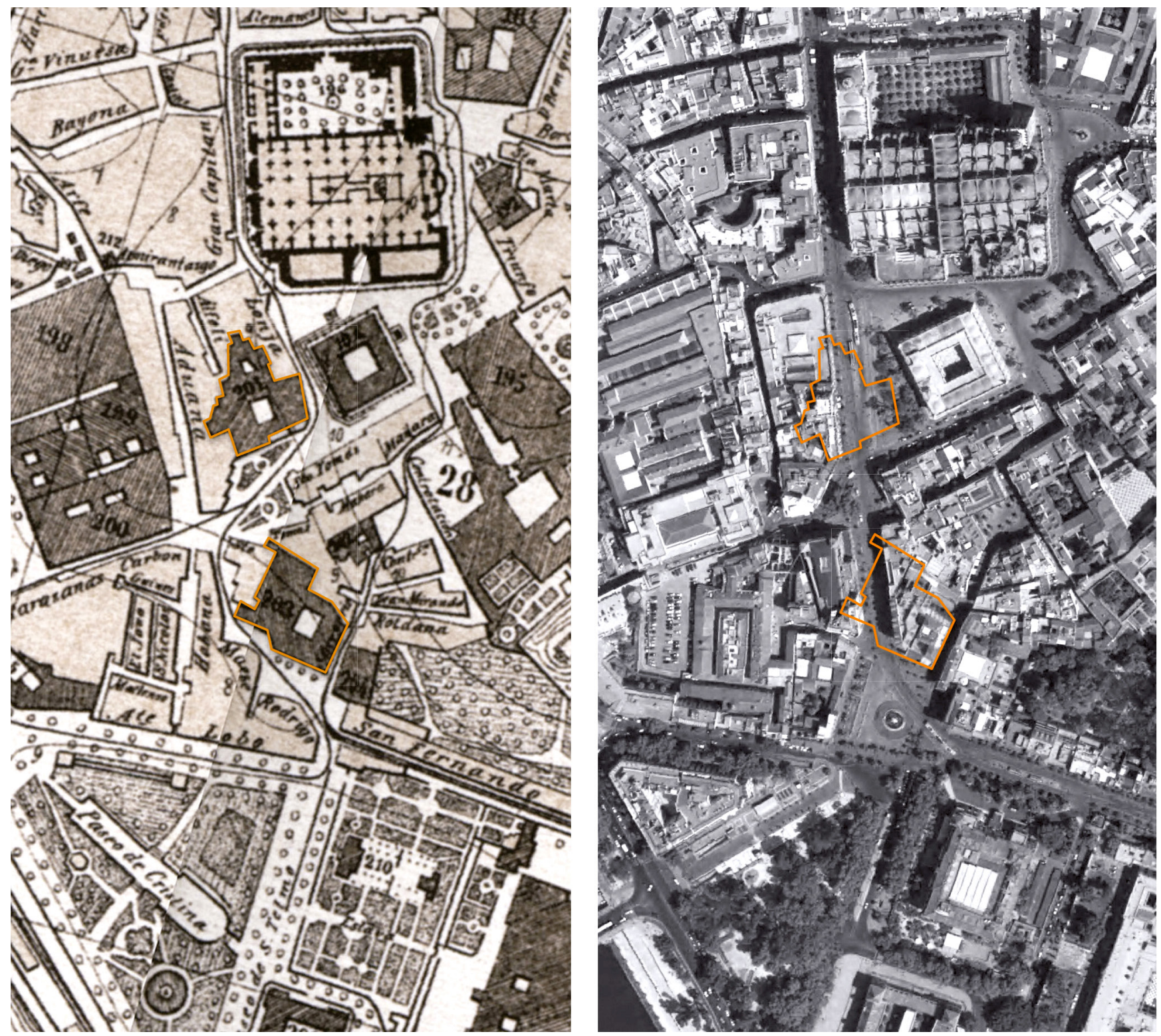

Figura 1. Comparación a la misma escala de un detalle del Plano de Sevilla de 1891, de Padura y de la Vega, y una ortofoto digital de 2010 del Instituto de Estadística y Cartografía de Andalucía, identificando las siluetas de los antiguos Colegios de Santa María de Jesús (demolido en 1909) y de Santo Tomás (demolido en 1927). Permite ilustrar la entidad de las piezas que se destruyeron para la apertura de estos dos tramos de Avenida.

a conseguir en la ciudad, están presentes en muchas de las breves reflexiones que acompañan a los documentos urbanísticos de la época ${ }^{1}$.

Esta voluntad de manipulación de la ciudad tomó forma gráfica en el Proyecto General de Reformas de la Capital de 1895, promovido por el Ayuntamiento y elaborado por el arquitecto municipal José Sáez López, en el que se propusieron una serie de intervenciones dirigidas a dotar a Sevilla de una nueva estructura viaria (12). El proyecto era relativamente posibilista, ya que aún dentro de la cultura urbanística dominante, en la mayoría de las aperturas que planteaba intentaba valorar la capacidad del viario existente para que, con un número limitado de operaciones, se consiguiese dotar a la ciudad de mayor permeabilidad, intentando evitar una imposición excesiva de ejes que pudieran resultar absolutamente ajenos a la realidad (Figura 3). Entre todas las intervenciones previstas, se dio prioridad a la actuación en el entorno de la Catedral para conseguir una comunicación directa entre el centro del poder civil, representado por las Casas Consistoriales, y los crecimientos meridionales vinculados al puerto fluvial y -poco después- al desarrollo de la Exposición Iberoamericana. La idea de la apertura de la Avenida formó parte no sólo del proyecto municipal de 1895, sino que estaba presente en otras propuestas que se presentaron en la ciudad a comienzos del xx, lo que implica que se trataba de una voluntad de considerable consenso entre los agentes que intervenían en la política urbanística local ${ }^{2}$.

La mayoría de los expedientes municipales de alineaciones del xix y principios del xx existentes en el Archivo Histórico Municipal de Sevilla (AHMS) contienen sucintas memorias, expresivas de la mentalidad higienista que intenta sanear una ciudad que se considera insalubre (10) (11). Tal como indica Manuel Trillo (1980), las propuestas presentadas por Miguel Sánchez-Dalp, el Conde de Halcón y el Conde de Colombí, contemplaban igualmente el gran eje de conexión entre el Ayuntamiento y la puerta de Jerez (13). 


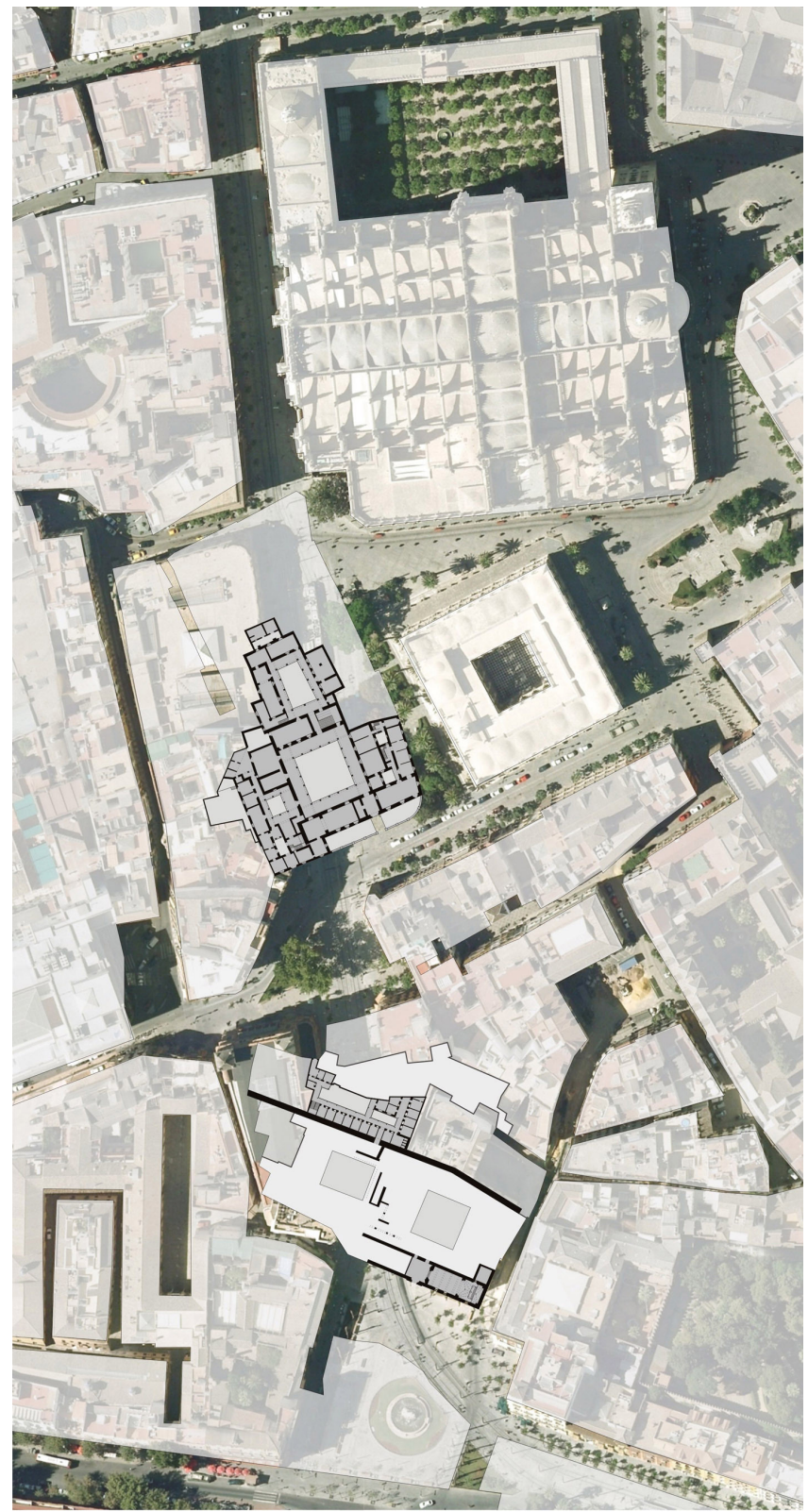

Figura 2. Fotomontaje de la planta del antiguo Colegio dominico de Santo Tomás y del antiguo Colegio de Santa María de Jesús sobre fotografía aérea de 2007. Se incorporan las trazas de las manzanas del entorno en 1906, los datos conocidos hasta la fecha de Santa María de Jesús y la planimetría militar redibujada del edificio de Santo Tomás, existente en el Archivo Militar Intermedio de Sevilla (AMIS). Dibujo: J. Tejido y F. Marín.

La creación de la Avenida se concretó mediante el citado proyecto de apertura de 1906, firmado por Sáez López como arquitecto municipal. El eje dibujado se fue realizando lentamente, con rectificaciones de trazado y ampliaciones de sección, ejecutado por tramos a lo largo de veinte años -hasta 1927-, concluyéndose muchos de los edificios de la nueva escena urbana bien entrada la década de los años treinta y algunas edificaciones frente a la Catedral a mediados del siglo xx. El objetivo prioritario de la intervención era abrir la vía, eliminando para ello todas aquellas piezas situadas en mitad del trazado, que pasaban a considerarse como obstáculos al denominado «ensanche de la población». Se confiaba en que el tamaño del nuevo espacio público y el porte de las arquitecturas que surgirían a continuación construirían la deseada Avenida de acceso al centro monumental de la ciudad. El proyec- to planteaba un horizonte de actuaciones por desarrollar, que precisaba de una instrumentación más detallada para cada tramo y debía desglosarse en expedientes concretos, que a su vez necesitaban de una política municipal de expropiación de los edificios destinados a ser demolidos. La primera versión de su trazado se basaba en el reconocimiento de la anchura del segmento colindante a la Catedral $-18,30 \mathrm{~m}$ - como medida adecuada para la definición del resto de la Avenida (Figura 4), planteando una alineación continua con esa dimensión para el resto de los tramos.

La comunicación directa de la plaza Nueva con los crecimientos meridionales de la ciudad obligaba a atravesar las manzanas situadas al sur de la Catedral, que se encontraban conformadas en relación a lienzos de muralla que habían constituido parte del Alcázar Viejo (14). Las piezas más importantes de dichas manzanas eran precisamente los antiguos Colegios de Santa María de Jesús y Santo Tomás. El primero había sido vendido en 1901 por el Arzobispado al promotor inmobiliario Juan Bautista Calvi, que vio una oportunidad de negocio en la adquisición de un inmueble a través del cual debía realizarse la gran vía (15). La superficie del edificio adquirido le garantizaba que incluso con la deducción del espacio de viario dispondría de solares edificables magníficamente situados (16). Las demoliciones en este tramo, promovidas en 1909 por Calvi, supusieron la desaparición de esta primera sede de la Universidad de Sevilla a excepción de la capilla, destruyéndose el conjunto originario del siglo xvI con todos los elementos que se habían ido añadiendo, como una librería y sala rectoral dieciochescas (17), y una ampliación del Seminario construida en el último tercio del xIx por el Arzobispado, con tipología de corral de vecinos (18). En este tramo, el trazado de la apertura se superpuso a la ciudad con gran indiferencia hacia la morfología subyacente. Prueba de ello es la planta de algunas de las parcelas que surgieron en esta zona, como la edificada por el arquitecto José Espiau y Muñoz para el promotor Calvi sobre los terrenos del antiguo Seminario, que presenta una enorme tensión geométrica en el centro del edificio, provocada por la imposición de la apertura sobre los antiguos límites de propiedad (Figura 5).

Al poco tiempo del inicio de los trabajos de apertura de la Avenida, en el extremo norte de la intervención se corrigió la dimensión inicial, aprobándose en 1912 una nueva sección más amplia, de 22,60 m. La lógica interna que subyacía en el proyecto, que consideraba prioritaria la continuidad del espacio resultante, se trasladó inmediatamente a los demás tramos, de tal manera que el segmento central de la Catedral -que había servido inicialmente para dimensionar la anchura de la operación- se redefinió con un nuevo cambio de alineaciones (19), adaptándolo a la medida definitiva de 22,60 m, también con proyecto de Sáez López (Figura 6). En este plano, la inflexión que aparece frente a la Catedral es la portada del antiguo Colegio de San Miguel, que se conservó -en su posición originalintegrada en la composición de fachada de un edificio regionalista realizado en 1927 por el arquitecto José Espiau y Muñoz (20), para lo cual la alineación aprobada se adaptó ligeramente con la intención de conservar este elemento.

La apertura del tramo de Santo Tomás, frente al Archivo de Indias, fue la más tardía en ejecutarse, por las arduas negociaciones entre el Ayuntamiento y el Estado para la cesión del edificio, que se utilizaba como Gobierno Militar y Comandancia de Artillería desde mediados del xIx (Figura 7). Era un conjunto articulado en torno a dos claustros porticados, con arquerías 


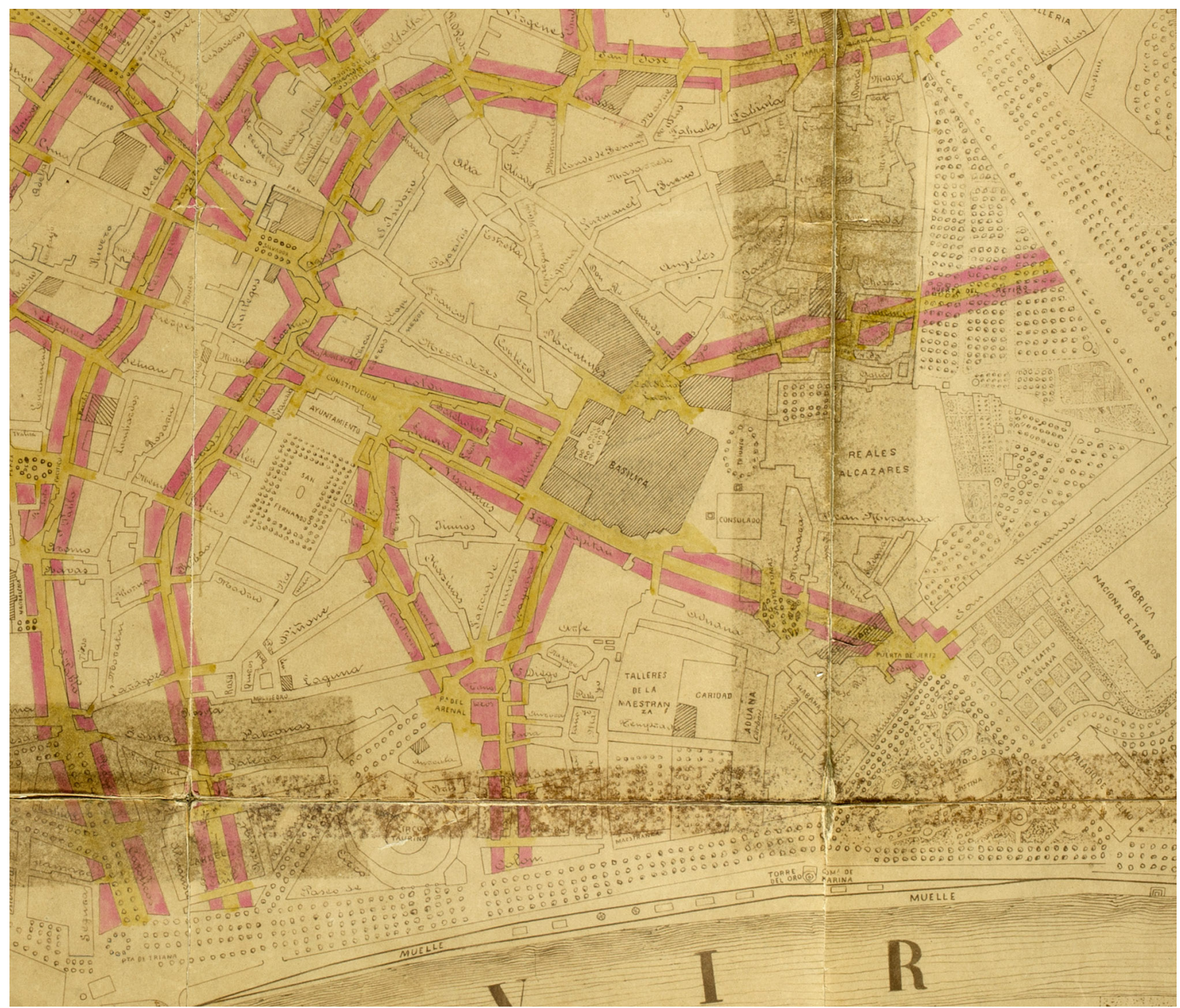

Figura 3. Proyecto General de Reformas en la Capital (1895). Detalle de la zona meridional del casco histórico. Arquitecto municipal José Sáez López. ( ICAS-SAHP. AHMS, Obras Públicas 133/1893. $1^{\text {a }}$ pieza).

sobre columnas, y otros patios menores. Una vez que el Estado lo cedió a la ciudad por decreto de 1926, su demolición se llevó a cabo por el Ayuntamiento en la primavera de 1927, mediante un concurso de demolición entre empresas del sector (21).

A partir de la demolición Santo Tomás, la apertura de la Avenida empezó a ser realidad. El plano de 1906, en base al cual se había desarrollado toda la operación de ensanche, había ido cumpliéndose poco a poco, reconsiderándose determinados encuentros con calles adyacentes. En junio de 1928, a la vez que se estaban definiendo las alineaciones definitivas de la manzana de Santo Tomás, se acometieron obras para la instalación de un jardín delantero al Archivo de Indias (Figura 8).

\section{ASPECTOS PATRIMONIALES DE LA APERTURA}

Además del tejido doméstico que desapareció en la zona norte de la intervención, las pérdidas patrimoniales más singulares se centraron en los dos edificios que desde su construcción -a principios del quinientos- hasta la época ilustrada centraron la vida universitaria de la ciudad, el
Colegio de Santa María de Jesús y el Colegio dominico de Santo Tomás, dos piezas de notable calidad arquitectónica, que tras los diversos avatares históricos por los que fueron pasando (que no excluyeron considerables procesos de deterioro), terminaron sus días convirtiéndose drásticamente en espacio público. Las circunstancias que fueron afectando a dichos conjuntos muestran un proceso de «destinos paralelos» entre ellos: ambos se concluyeron en la segunda década del siglo XVI, como piezas importantes de la zona meridional del recinto intramuros, ambos se crearon para albergar el uso residencial y docente de un colegio-universidad (22) y ambos fueron afectados por la apertura de la Avenida, con pocos años de diferencia. Podemos incluso afirmar que los dos edificios fueron valorados a lo largo del XIX como deseables tanto por el Ejército como por el Arzobispado de Sevilla, presentando para ambas instituciones ciertas semejanzas en sus capacidades funcionales (23). Estas apreciaciones ponen de manifiesto no sólo la posición urbana privilegiada de ambos inmuebles, sino también la flexibilidad funcional que ofrecían, al estar organizados en torno a varios claustros que combinaban estancias de mayor tamaño en planta baja, 


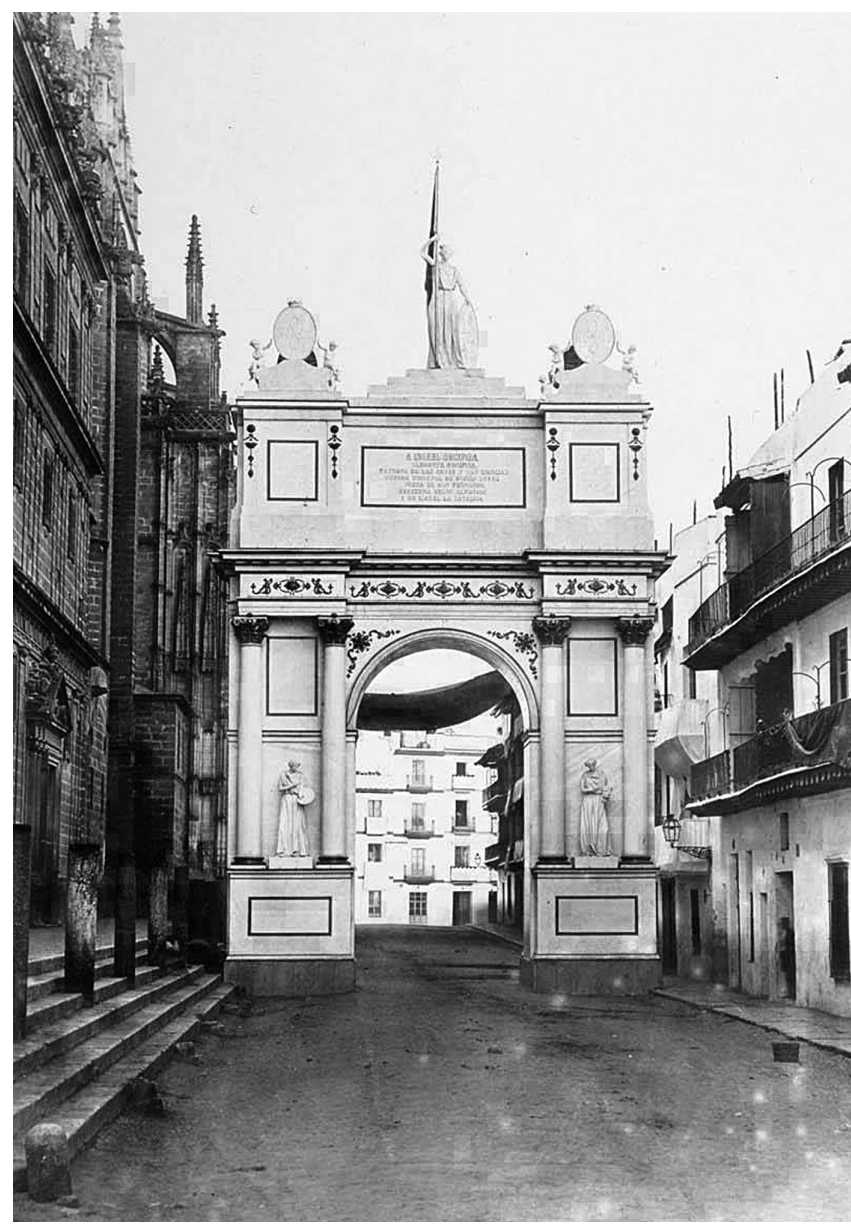

Figura 4. Sevilla, arco en las gradas de la catedral (1862). (Fotografía de Charles Clifford. Incluida en el Álbum de Recuerdos fotográficos de la visita de SS.MM. y AA.RR. á las provincias de Andalucía y Murcia en Setiembre y Octubre de 1862). La sección de este tramo se tomó inicialmente como medida a extender al resto del proyecto. originariamente dedicadas a aulas y espacios académicos singulares, con cámaras de menor escala para residentes, ubicadas en su mayoría en los niveles de planta principal. Dicho esquema tipológico permitió la adecuación de los dos conjuntos a los usos que cada uno de ellos fue recibiendo sin necesidad de grandes transformaciones, facilitando la utilización de Santo Tomás como Gobierno Militar y de Santa María de Jesús como Seminario Conciliar.

Como excepción a ese prolongado paralelismo, la desaparición de Santo Tomás no dejó como pervivencia ningún espacio arquitectónico, como sí sucedió con la Capilla de Santa María de Jesús, que se mantiene. Del antiguo Colegio dominico tan sólo se han conservado elementos constructivos reutilizados en otros edificios: un alfarje o forjado plano de madera labrada y decorada instalado en 1875 en el Salón Santo Tomás del Ayuntamiento (24) y el artesonado de la capilla colegial, que en 1890 se instaló transformado (convertido en plano horizontal decorativo) en la nave del Lagarto de la Catedral (25), donde ha permanecido hasta mediados de los años ochenta del siglo xx, en que ha sido desmontado. De Santa María de Jesús, además de la capilla, se ha conservado la parte inferior de la portada colegial, que se trasladó al compás del convento de Santa Clara y fue montada por Juan Talavera y Heredia en 1924 como acceso al Museo Arqueológico municipal de la Torre de Don Fadrique, donde permanece.

A estas consideraciones debe añadirse que el primer trazado de Sáez López para la Avenida hubiera significado también la destrucción de la capilla de Santa María de Jesús -a pesar de haber sido declarada monumento nacional en 1901- y habría implicado la demolición de otros dos elementos históricos de alto valor patrimonial, la torre de Abd-el Aziz y el arco de la Plata (Figura 9). Estas piezas pudieron conservarse porque el Consistorio fue advertido de las consecuencias de dicho trazado por el concejal responsable de alineaciones, y se ordenó al arquitecto municipal su modificación para respetarlas (26).

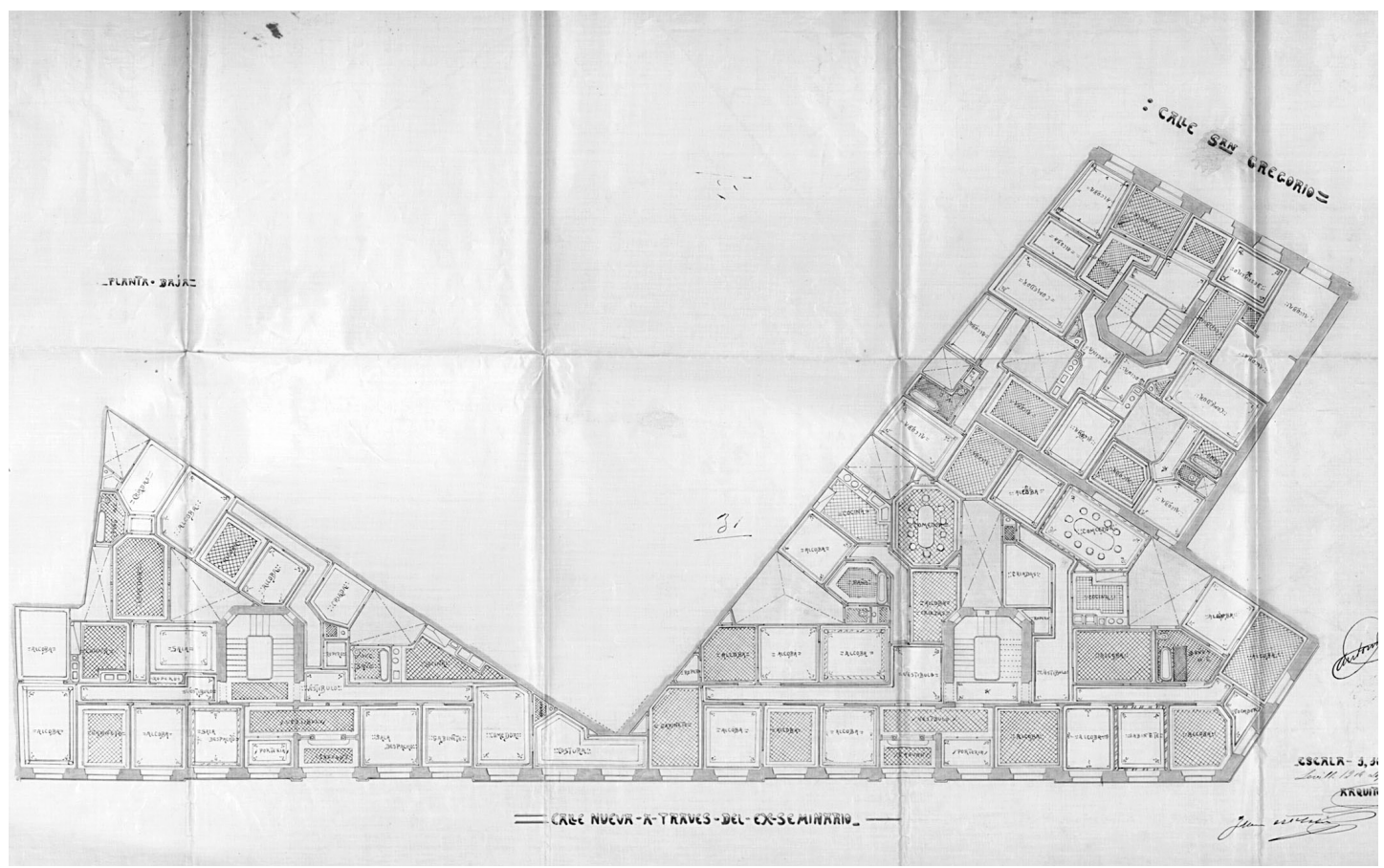

Figura 5. Planta de edificio de viviendas en calle nueva a través del Ex-Seminario (1910). Arquitecto José Espiau y Muñoz. ( ${ }^{(}$ICAS-SAHP. AHMS. Construcciones y Reconstrucciones. 2-3-50). 


\section{TENTATIVAS DE NUEVAS APERTURAS}

La técnica urbanística de las grandes demoliciones para crear nuevos espacios públicos tuvo en este mismo entorno un intento de reedición tras la guerra civil. En 1940, con la apertura de la Avenida completamente consolidada, el Ayuntamiento inició los trámites de una intervención consistente en la creación de una plaza frente a la Catedral, proyecto que se encargó al arquitecto municipal Juan Talavera y Heredia, que no llegó a ejecutarse (27). La ordenación, que implicaba la destrucción del antiguo Colegio de San Miguel, intentaba crear un gran espacio público para enmarcar y dar mayor visibilidad al conjunto formado por las tres puertas de los pies del templo metropolitano, presididas por la portada mayor de

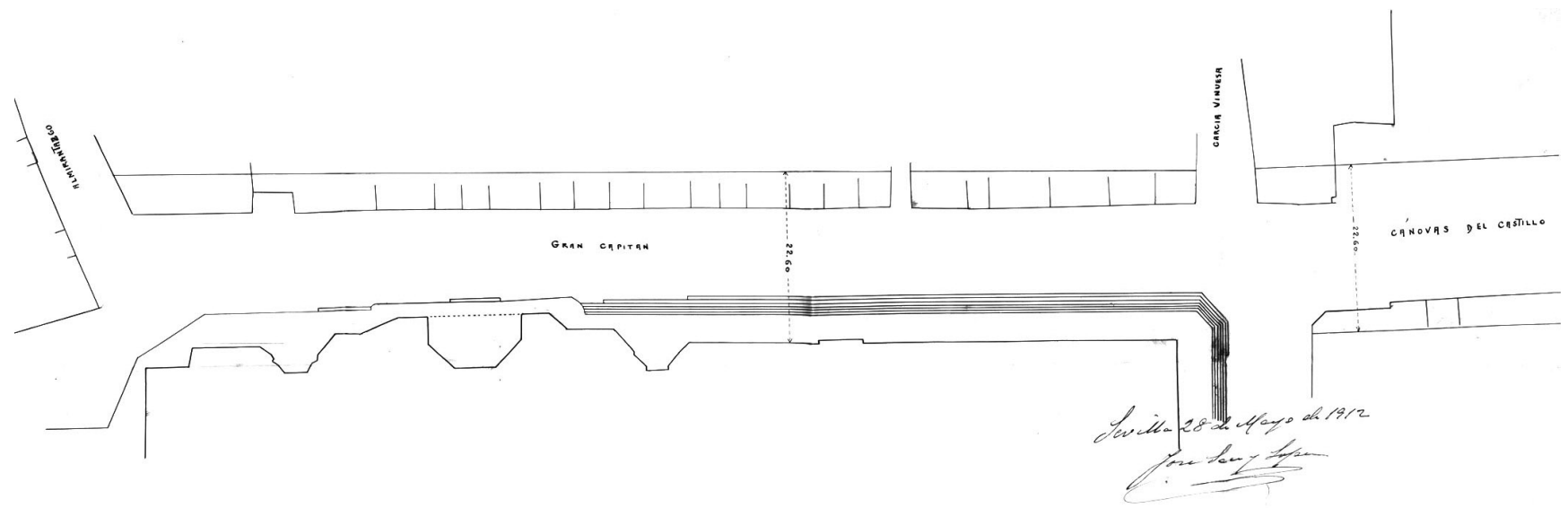

Figura 6. Proyecto de alineación de la calle Gran Capitán (1912). Arquitecto municipal José Sáez López.

๑ ICAS-SAHP. AHMS. (Colección Alfabética. Alineaciones. caja 41. expte.413).

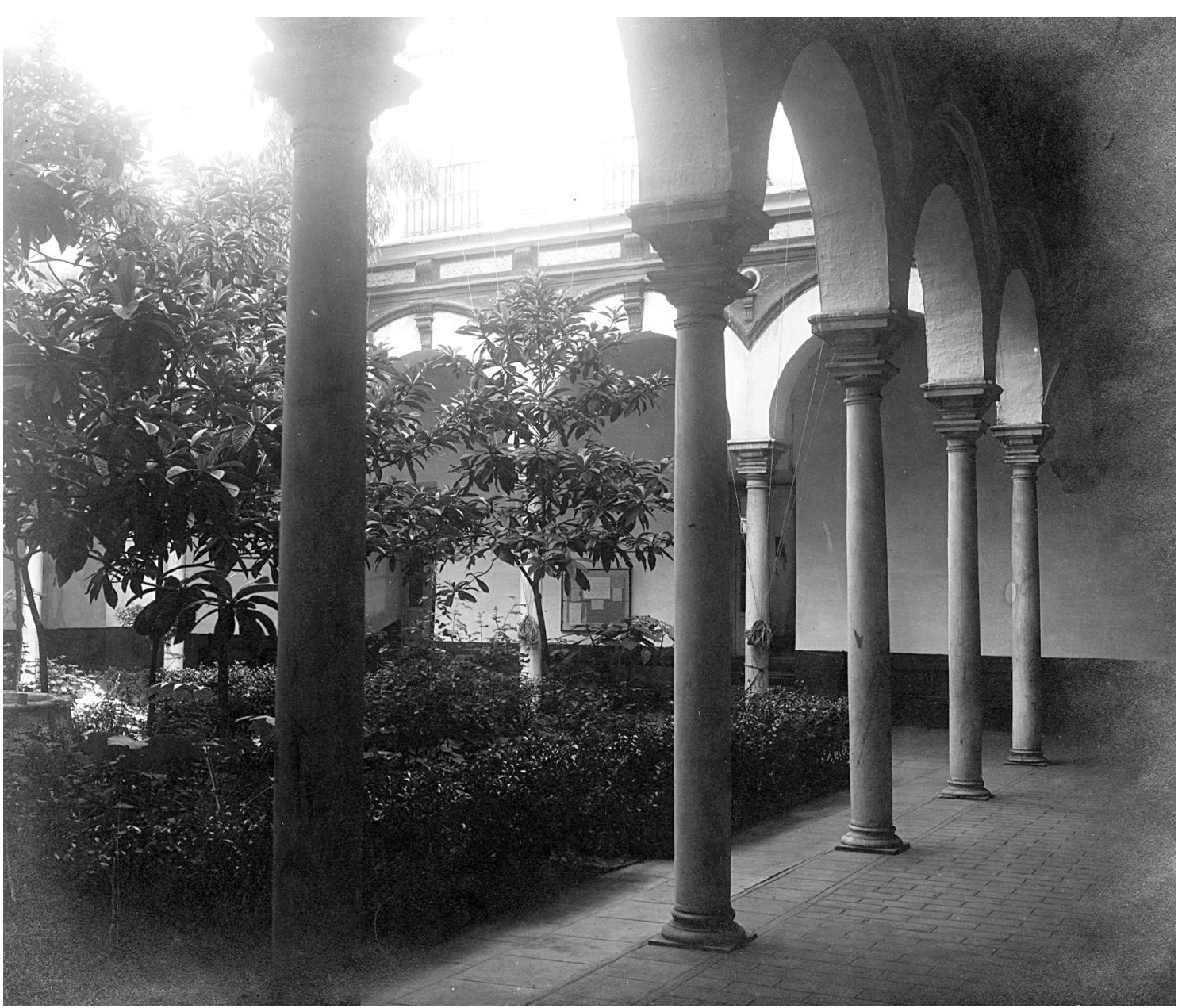

Figura 7. Claustro principal del antiguo Colegio de Santo Tomás. (Fotografía de José María González-Nandín y Paul. Año 1925. Fototeca del Laboratorio de Arte de la Universidad de Sevilla). 


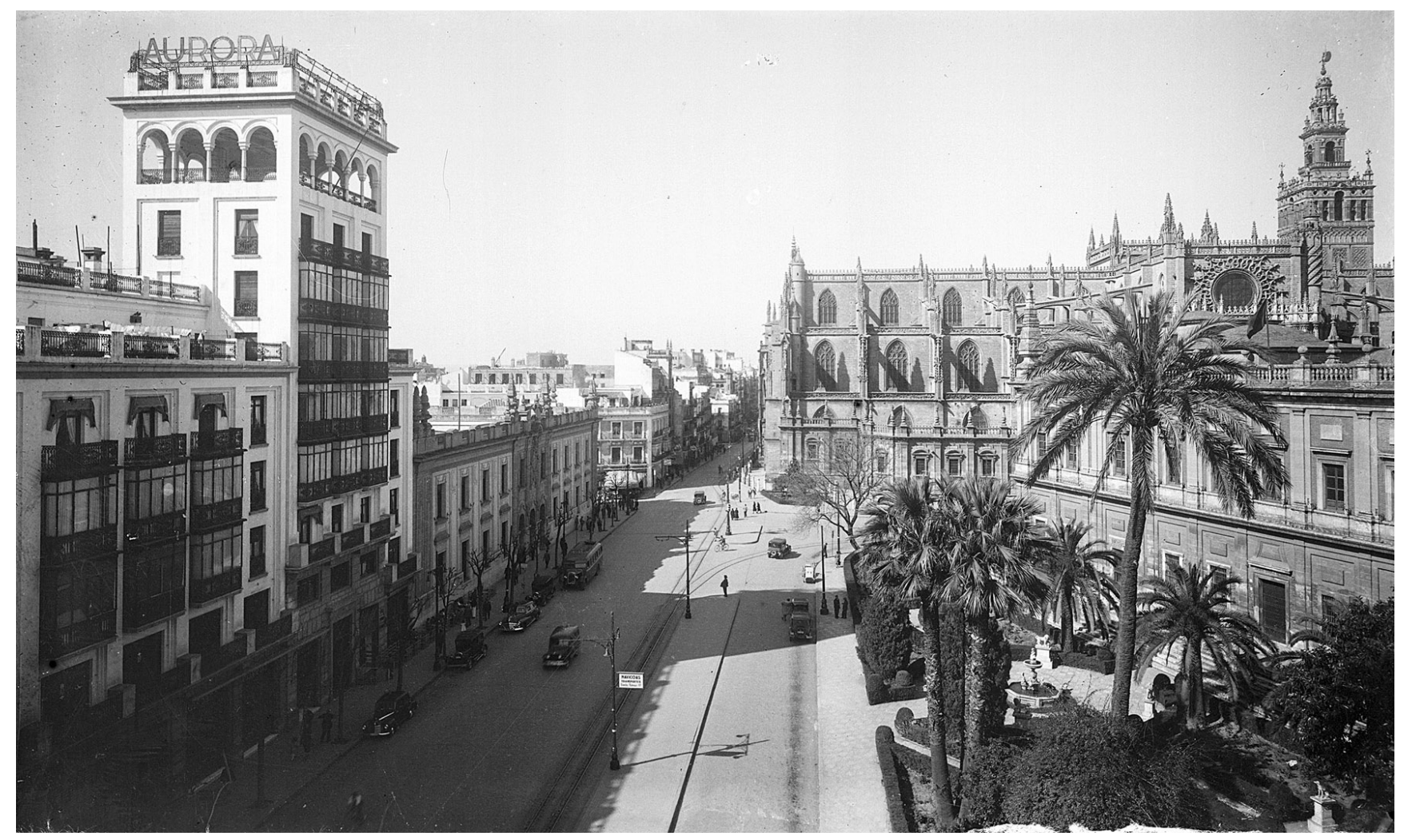

Figura 8. Vista general de la Avenida hacia 1937. (Colección Roisin. Instituto de Estudios Fotográficos de Cataluña. Ref. ACM-9-26913v. ๑ Lucien Roisin, VEGAP, Sevilla, 2016).
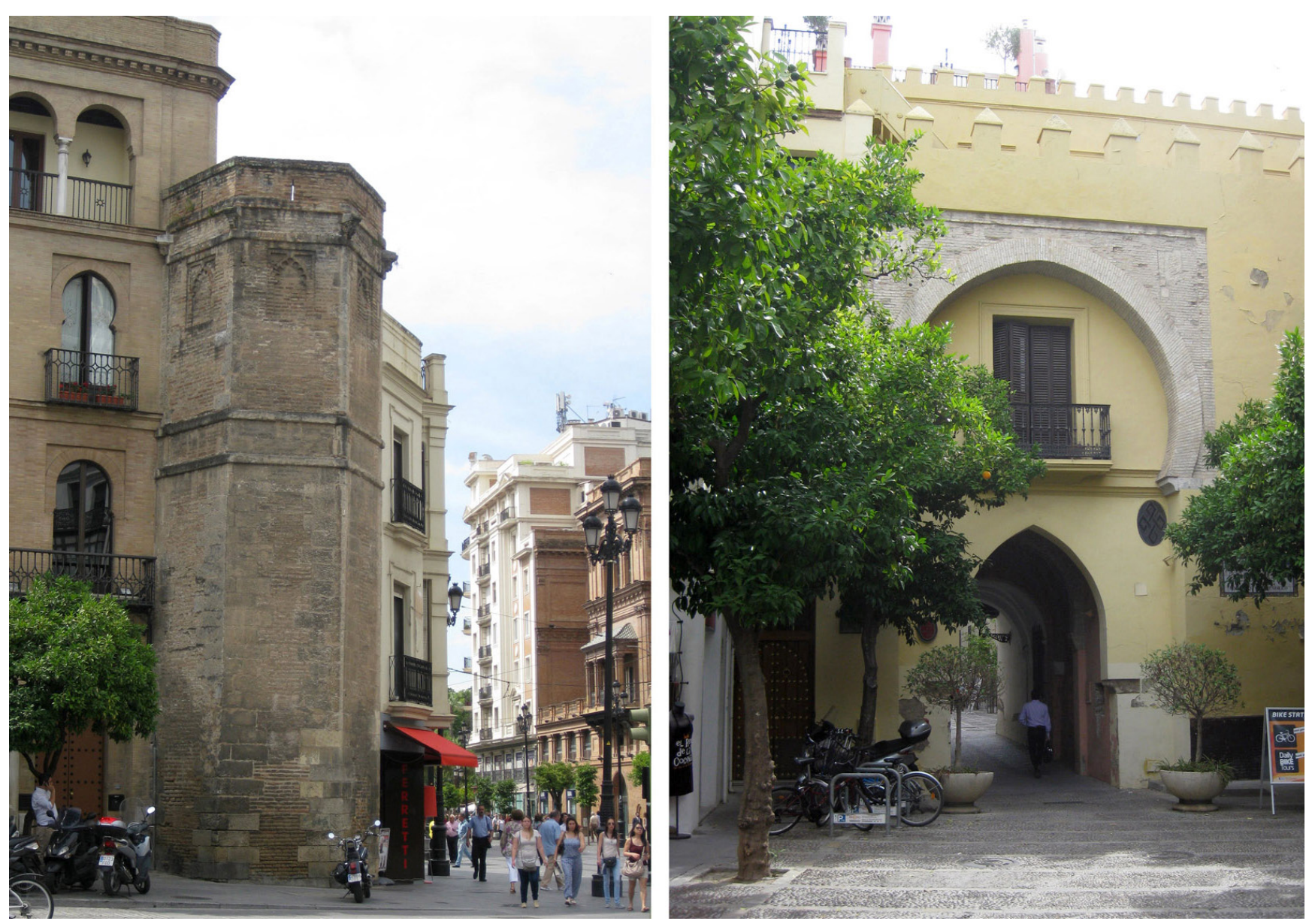

Figura 9. Torre de Abd-el Aziz (siglo xiI) y Arco de la Plata (siglo xiII). (Fotos del autor, 2013). 

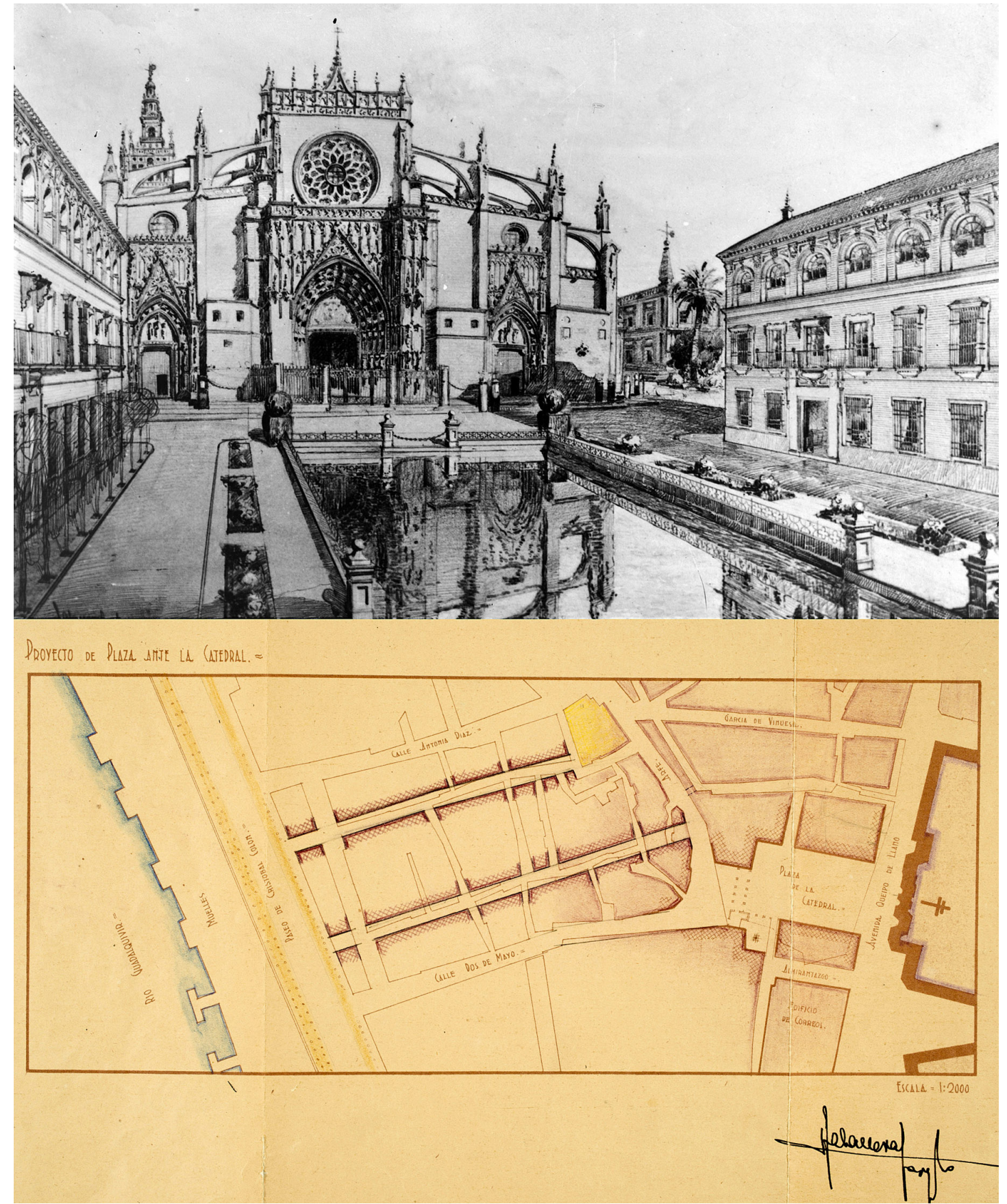

Figura 10. Perspectiva y planta urbana del Proyecto de Plaza frente a la Catedral (1940). Arquitecto municipal Juan Talavera y Heredia. ( ${ }^{\odot}$ ICAS-SAHP. AHMS. Obras Públicas. Expte. 78/1940).

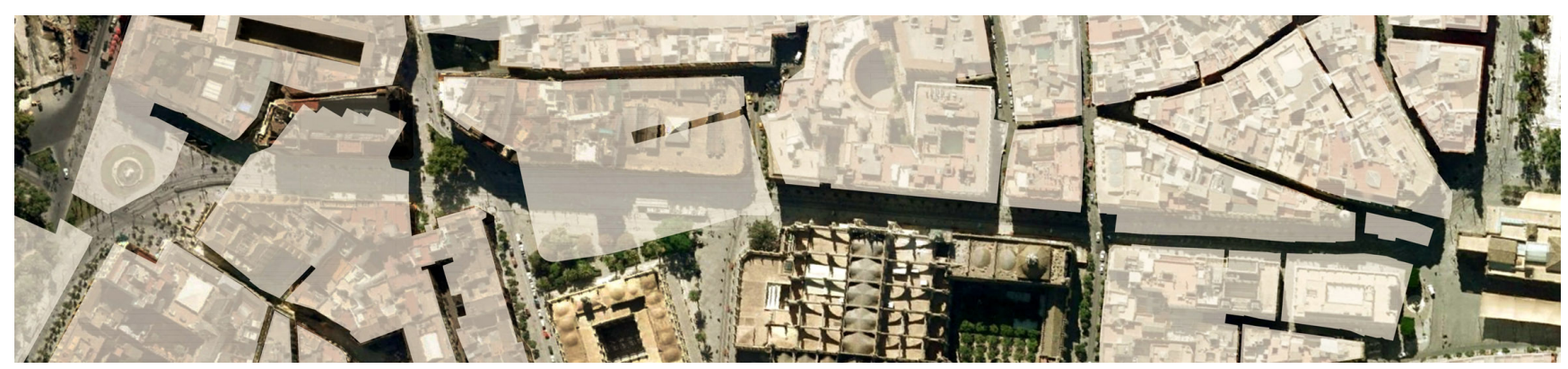

Figura 11. Fotomontaje de la trama urbana de 1906 sobre ortofoto de Sevilla del año 2007. La expresión gráfica mediante transparencia de las manzanas existentes a comienzos del siglo xx permite comprobar la envergadura de la operación de apertura de la Avenida. Dibujo: J. Tejido y E. Nieto. 
la Asunción, el gran rosetón de la nave central y el grupo de arbotantes. Talavera propuso la utilización de una lámina de agua como elemento duplicador de la imagen de la arquitectura que se deseaba subrayar, al igual que había hecho quince años antes en el ámbito mucho más recogido de la Torre de Don Fadrique. La plaza se definía con una envolvente ecléctica de control formal del espacio, que mediante un pórtico abierto se intentaba articular con algunas preexistencias que proponía conservar, como el postigo del Aceite. La apertura iba acompañada de una reforma urbana de ensanche en el adyacente barrio de la Carretería, dirigida a conseguir una mayor permeabilidad hacia el río, en sentido este-oeste (Figura 10). Se planteaba la ampliación de las calles que se dirigían al antiguo puerto, intentando facilitar la conexión entre el espacio fluvial y la Avenida. La iniciativa se abandonó en 1944 y se retomó diez años después, con proyecto del arquitecto municipal Luis Gómez Estern sobre la base planteada por Talavera, que tampoco se ejecutó.

\section{CONCLUSIONES}

La apertura de la Avenida de Sevilla es una operación urbana ejecutada en el primer tercio del siglo xx. Su realización se sustentó en una confianza absoluta en la posibilidad de que la ciudad resultaba mejorable y debía ser urgentemente transformada, dedicándose a ello amplios sectores de la sociedad con denodado esfuerzo, aún a riesgo de dejar en el camino elementos de valor. La clave de la violencia con que se impuso el trazado en la zona sur de la intervención se encuentra en que este sector de la ciudad, destinado a necesitar de una gran permeabilidad viaria por el desarrollo hacia el sur que implicaba la Exposición Iberoamericana, era inicialmente un recinto defensivo del Alcázar, y por lo tanto, originariamente planteado como una contención -la antítesis de un espacio de conexión-, de tal manera que las construcciones establecidas sobre dicho recinto, apoyadas en el trazado de sus muros, precisaron ser fuertemente manipuladas. Dado que su morfología se apoyaba en un recinto «transversal» a la direccionalidad de la apertura, no se prestaba a recibir la pretendida reforma urbana con intervenciones ligeras o de menor calado. De este modo, los objetos arquitectónicos no tuvieron la posibilidad de una transformación moderada para ofrecer una solución de apertura viaria que evitase su destrucción (Figura 11). Desde esta posición conceptual, se generó una potente metamorfosis urbana, pasándose de una trama doméstica abigarrada, con un viario estrecho y serpenteante, a un espacio público de mayor escala, que ofrecía la «gran perspectiva» como recurso para ensalzar los edificios de relevancia, la Catedral y el Archivo de Indias, que de ser elementos por descubrir pasaron a actuar como referentes visuales autónomos, comportándose como objetos abstractos de una morfología constituida casi sin caserío, sólo por monumentos.

\section{REFERENCIAS}

(1) Proyecto de alineación para las calles Cánovas del Castillo, Gran Capitán, Cardenal González, Santo Tomás, Maese Rodrigo y Jerez. (1906). En Colección Alfabética. Alineaciones, caja 36. expte. 343. Sevilla: Archivo Histórico Municipal de Sevilla (AHMS).

(2) Collantes de Terán Sánchez, et al. (1993). voz «Constitución, Avenida de la». En Diccionario histórico de las calles de Sevilla, vol. I, (pp. 229-237). Sevilla: Consejería de Obras Públicas y Transportes y Ayuntamiento de Sevilla.

(3) Monclús Fraga, J., et al. (2011). La Gran Vía de Zaragoza y otras grandes vías. Catálogo de la exposición. Madrid: Ministerio de Vivienda y Editorial Lampreave.

(4) Villar Movellán, A. (1979). Arquitectura del regionalismo en Sevilla: 190o-1935. Sevilla: Diputación Provincial.

(5) AHMS. Colección Alfabética. Alineaciones. caja 42 expte. 432/1915 -caja 33 expte. 256(4)/1926- caja 42 expte. 422. Sevilla: Archivo Histórico Municipal de Sevilla (AHMS).

(6) Expediente formado para reconstruir el antiguo Seminario Conciliar. (1909). Construcciones y Reconstrucciones. expte. 2-3-50. Sevilla: Archivo Histórico Municipal de Sevilla (AHMS).

(7) Seminario Conciliar. Expediente sobre reparación extraordinaria y ampliación del edificio. (1863). Arquitecto Manuel Portillo Navarrete. Administración. Casas parroquiales y reconstrucciones de templos. Legajo 14545. expte. 4. Archivo General del Arzobispado de Sevilla (AGAS).

(8) Hunt Ortiz, M. (2008). Memoria Científica final de la Intervención Arqueológica preventiva del Metro Ligero Centro «Metrocentro». Archivo de la Delegación Provincial de la Consejería de Cultura de la Junta de Andalucía.

(9) Suárez Garmendia, J. M. (1986). Arquitectura y Urbanismo en la Sevilla del siglo XIX. Sevilla: Diputación Provincial.

(10) Hauser, Ph. (1882). Estudios médico-topográficos de Sevilla. Acompañados de un plano sanitario-demográfico y 70 cuadros estadísticos. Sevilla: Librería de Tomás Sanz.

(11) Hauser, Ph. (1884). Estudios médico-sociales de Sevilla. Acompañados de 90 cuadros estadísticos. Sevilla: Librería de Tomás Sanz.

(12) Proyecto General de Reformas en la Capital. (1893). Obras Públicas. Expte. 133/1893. $1^{a}$ pieza. Sevilla: Archivo Histórico Municipal de Sevilla (AHMS).

(13) Trillo de Leyva, M. (1980). La Exposición Iberoamericana. La transformación urbana de Sevilla. Sevilla: Ayuntamiento de Sevilla.

(14) Jiménez Martín, A. (1981). La arquitectura de nuestra ciudad. Sevilla: Colegio Oficial de Aparejadores y Arquitectos Técnicos.

(15) Espiau Eizaguirre, M. (1991). La Casa de la Moneda de Sevilla y su entorno: historia y morfología. Sevilla: Universidad de Sevilla y Consejería de Obras Públicas y Transportes.

(16) Expediente formado con motivo de la ejecución de obras en parte de lo que fue Seminario Conciliar. (1909). Obras Públicas 95/1909. Sevilla: Archivo Histórico Municipal de Sevilla (AHMS).

(17) Obra de la librería. (1742-1756). legajo 609.21 a). Sevilla: Archivo Histórico de la Universidad de Sevilla (AHUS).

(18) Proyecto de ampliación del Seminario Conciliar. (1863). Administración. Casas parroquiales y reconstrucciones de templos. leg. 14545. expte. 4. Archivo General del Arzobispado de Sevilla (AGAS). 
(19) Proyecto de alineación de la calle Gran Capitán. (1912). C. A. Alineaciones. caja 41. expte. 413. Sevilla: Archivo Histórico Municipal de Sevilla (AHMS).

(20) Proyecto de reconstrucción de la casa ${ }^{\circ} 46$ de la Calle Gran Capitán. (1927, 27 de julio). Arquitecto José Espiau y Muñoz. Construcciones y Reconstrucciones 8-144. c/Gran Capitán no 46 años 1926-31. Sevilla: Archivo Histórico Municipal de Sevilla (AHMS).

(21) Expediente formado para convocar concurso de derribo del edificio ex-Convento de Santo Tomás. (1927). Obras Públicas. 1927/1.1. Sevilla: Archivo Histórico Municipal de Sevilla (AHMS).

(22) Aguilar Piñal, F. (1991). Historia de la Universidad de Sevilla. Sevilla: Secretariado de publicaciones de la Universidad de Sevilla.

(23) Exconvento de Santo Tomás. Devolución al Arzobispado. $2^{a}$ División Orgánica. 1859-1863. caja 1350. carpeta 5. Sevilla: Archivo Militar Intermedio de Sevilla (AMIS).

(24) Fernández Martín, M. (1992). El Patrimonio mueble. En AA.VV. Ayuntamiento de Sevilla. Historia y patrimonio. Sevilla: Guadalquivir.

(25) Santos y Olivera, B. (1930). Guía ilustrada de la catedral de Sevilla. Madrid: Talleres Tipográficos Voluntad.

(26) Alineaciones. Colección Alfabética, caja 36, expte. 343. leg. 8 del expte. Sevilla: Archivo Histórico Municipal de Sevilla (AHMS).

(27) Proyecto de Plaza frente a la Catedral. (1940). Arquitecto municipal Juan Talavera y Heredia. Negociado de Obras Públicas. Expte. 78/1940. Incluido en el Expte. 18/1954. Sevilla: Archivo Histórico Municipal de Sevilla (AHMS). 